\title{
Transplantation of neural stem cells preconditioned with high-mobility group box 1 facilitates functional recovery after spinal cord injury in rats
}

\author{
XIN XUE $^{1}$, LIANG ZHANG ${ }^{1}$, XIANG YIN $^{1}$, XING-XING CHEN $^{2}$, ZONG-FENG CHEN $^{1}$, \\ CHEN-XU WANG ${ }^{1}$, YU XIANG ${ }^{1}$, MING-YONG LIU ${ }^{1}$ and JIAN-HUA ZHAO ${ }^{1}$ \\ ${ }^{1}$ Department of Spinal Surgery, Daping Hospital, Research Institute of Surgery, \\ Third Military Medical University (Army Medical University), Chongqing 400042; ${ }^{2}$ Department of Orthopedic, \\ No. 517 Hospital of People's Liberation Army, Xinzhou, Shanxi 030002, P.R. China
}

Received December 21, 2019; Accepted July 6, 2020

DOI: $10.3892 / \mathrm{mmr} .2020 .11565$

\begin{abstract}
Spinal cord injury (SCI) is a devastating disorder that often results in temporary and/or permanent functional impairment below the injured level. To date, few satisfactory therapeutic strategies are available to treat SCI. Hence, exploring novel strategies for $\mathrm{SCI}$ is an essential public health concern. Cell transplantation therapy, which is associated with neuroprotection, immunomodulation, axon regeneration, neuronal relay formation and myelin regeneration, provides a promising therapeutic strategy for SCI. The neuronal stem cell (NSC) preconditioning method is an emerging approach, which facilitates NSC survival and neuronal differentiation after implantation. The aim of the present study was to develop a feasible candidate for cell-based therapy following $\mathrm{SCI}$ in rats and to investigate the role of high mobility group box-1 (HMGB1) in NSC activation. The results of the present study showed that transplantation of NSCs, preconditioned with $1 \mathrm{ng} / \mathrm{ml}$ HMGB1, facilitated functional improvement of injured spinal cords, as indicated by Basso, Beattie and Bresnahan mean scores, mechanical hypersensitivity and cold stimulation. Meanwhile, the histological examination of hematoxylin and eosin staining indicated that engraftment of HMGB1-preconditioned NSCs resulted in decreased atrophy of the injured spinal cord. Meanwhile, the transplantation of HMGB1-preconditioned NSCs resulted in an increased number of functional Nissl bodies in neurons, as detected by Nissl staining, and an increase in the number of $\beta$ III-tubulin ${ }^{+}$
\end{abstract}

Correspondence to: Dr Ming-Yong Liu or Professor Jian-Hua Zhao, Department of Spinal Surgery, Daping Hospital, Research Institute of Surgery, Third Military Medical University (Army Medical University), 10 Yangzi River Road, Daping, Yuzhong, Chongqing 400042, P.R. China

E-mail: mingyong_liu@163.com

E-mail: zhaojianhua1964@yahoo.com

Key words: spinal cord injury, neural stem cells, high mobility group box-1, ERK signaling pathway, differentiation cells in the epicenter of injured spinal cords in rats with SCI. In addition, the results also demonstrated that $1 \mathrm{ng} / \mathrm{ml}$ HMGB1 promoted the differentiation of NSCs into neurons, and that the ERK signaling pathway played an important role in this process. In conclusion, the present data indicated that the preconditioning strategy with $1 \mathrm{ng} / \mathrm{ml}$ HMGB1 may present a feasible candidate for cell-based therapy following SCI in rats, which may enlarge the scope of HMGB1 in NSC activation.

\section{Introduction}

Spinal cord injury (SCI) is a devastating disease that often results in temporary and/or permanent functional impairment below the injured level $(1,2)$. Currently, strategies used to treat SCI, such as surgery with medical strategy, are supplemented with physical rehabilitation and/or gene therapy. Although these remedies provide some benefits, most patients with SCI are unable to achieve any substantial functional recovery (3). Considering that few satisfactory therapeutic strategies are available for SCI treatment (4), exploring new strategies for $\mathrm{SCI}$ is a cardinal public health issue.

Cell transplantation therapy is associated with immunomodulation, axon regeneration, neuroprotection, neuronal myelin regeneration and relay formation, thus making it a promising therapeutic strategy for SCI (5). Studies have indicated that neural stem cell (NSC) transplantation into lesions after SCI helps to regenerate injured tissue by promoting axonal regeneration, spinal conductivity and functional recovery (6-9). However, studies have also demonstrated that limited numbers of transplanted NSCs survive for the long-term in lesions (10-12). Thus, developing new approaches to improve NSC survival after engraftment is an important area of study when investigating cell-based strategies.

The NSC preconditioning method is an emerging approach that facilitates NSC survival and neuronal differentiation following implantation. Recently, it was revealed that NSC transplantation prior to hypoxia preconditioning promotes functional recovery, by enhancing the secretion of neurotrophic and growth factors [neurotrophin-3 (NT-3), glial cell line-derived neurotrophic factor (GDNF) and brain-derived 
neurotrophic factor (BDNF)] (2). The release of such factors increases the number of residual neurons, particularly 5-hydroxytryptamine- and choline acetyltransferase-positive neurons, and reduces the area positive for glial fibrillary acidic protein at the epicenter of the injured spinal cord in rats (2). Most recently, a previous study indicated that $1 \mathrm{ng} / \mathrm{ml}$ high mobility group box-1 protein (HMGB1) facilitates NSC proliferation and migration, via the HMGB1/advanced glycation end products (RAGE) axis to enhance filopodia formation in vitro (13). Hence, the effect of HMGB1 preconditioning prior to NSC transplantation on the functional recovery of injured spinal cords in rats, and the potential underlying mechanism need to be elucidated. The aim of the present study was to develop a cell-based approach for the treatment of SCI and to examine the role of HMGB1 in NSC activation.

\section{Materials and methods}

Animals. This study was approved by The Third Military Medical University Ethics Committee (approval no. SYXK 2012-0002) and followed the regulations of the China Laboratory Animal Guidelines (RB/T 019-2019 Edition). Every effort was made to minimize the number of animals used and their suffering. All Sprague Dawley (SD) rats were maintained on a constant photoperiod (12-h light/dark cycle), temperature $\left(22-25^{\circ} \mathrm{C}\right)$ and moisture (55-60\%), and provided food and water ad libitum. A total of 60 male SD rats (51 rats were used for research and 9 rats died during the experiment) and 8 embryonic day 14.5 (E14.5) SD rats were used in the present study. All rats were sacrificed by decapitation after anesthesia with $2 \%$ isoflurane/air mixture (1-2 1/min).

Primary NSC culture. A total of 8 E14.5 SD rats were employed to obtain primary NSCs. Briefly, the neocortices of pups were obtained and used for NSC culture after decapitation of E14.5 SD rats, as previously described (14). Then, the samples were washed twice in Dulbecco's modified Eagle's medium (DMEM; Gibco; Thermo Fisher Scientific, Inc.) with $10 \%$ fetal bovine serum (FBS; Gibco; Thermo Fisher Scientific, Inc.) after incubation in $0.25 \%$ trypsin-EDTA (Gibco; Thermo Fisher Scientific, Inc.) at $37^{\circ} \mathrm{C}$ for $30 \mathrm{~min}$. Thereafter, the tissue samples were triturated using a Pasteur pipette and passed through a 100- $\mu$ m nylon cell strainer (BD Falcon $^{\mathrm{TM}} ; \mathrm{BD}$ Biosciences) to collect the dissociated cells in the suspension, after washing twice with DMEM. Cell suspension was cultured in DMEM/F12 medium (Gibco; Thermo Fisher Scientific, Inc.) supplemented with B27 (Gibco; Thermo Fisher Scientific, Inc.), $20 \mathrm{ng} / \mathrm{ml}$ epidermal growth factor (PeproTech, Inc.), $20 \mathrm{ng} / \mathrm{ml}$ fibroblast growth factor 2 (PeproTech, Inc.) and $1 \%$ penicillin-streptomycin ( $\mathrm{vol} / \mathrm{vol}$; Beyotime Institute of Biotechnology) at $37^{\circ} \mathrm{C}$ in a humidified atmosphere with $5 \% \mathrm{CO}_{2}$, as recommended. For cell passaging, neurospheres were harvested by centrifugation $(100 \mathrm{x} \mathrm{g})$ at room temperature for $5 \mathrm{~min}$, dissociated in StemPro ${ }^{\mathrm{TM}}$ Accutase $^{\mathrm{TM}}$ Cell Dissociation reagent (Gibco; Thermo Fisher Scientific, Inc.) and cultured in medium as aforementioned. For HMGB1 preconditioning, NSCs were incubated in $1 \mathrm{ng} / \mathrm{ml} \mathrm{HMGB1}$ for $24 \mathrm{~h}$ as previously described (13), and NSCs was washed twice with DMEM before transplantation. NSCs at passage 3-5 were used for all experiments in the present study.
Surgical procedures. The surgical procedures were performed as previously described $(2,15)$. Briefly, 2-month old male SD rats (weight, 220-250 g) were placed in a stereotaxic frame after anesthesia with $2 \%$ isoflurane/air mixture (1-2 $1 / \mathrm{min})$. A 4-cm-long incision was made in the skin along the midline of the back, and a laminectomy was carried out to expose the thoracic 9-11 spinal segments, leaving the dura intact. Then, the spinal cord was compressed using a calibrated aneurysm clip, which provided $20 \mathrm{~g} / \mathrm{cm}^{2}$ pressure. The clip was released after $60 \mathrm{sec}$. Subsequently, the muscles and skin were sutured in separate layers $10 \mathrm{~min}$ after injection. Body temperature was maintained at $37 \pm 0.3^{\circ} \mathrm{C}$ by a feedback-controlled heating pad during surgery. Their bladders were emptied twice daily until they could do so themselves.

NSC transplantation. Following surgery, a total of 51 male SD rats ( $\mathrm{n}=17$ for each group) were randomly divided into three experimental groups: i) Control group, DMEM only; ii) NSC group, DMEM and NSCs; iii) HGMB1-NSC group, DMEM and HMGB1-preconditioned NSCs. The control group received $3 \mu \mathrm{l}$ DMEM, which was injected into the middle of the lesions. The NSC group received $2 \times 10^{5}$ normal NSCs in $3 \mu 1$ DMEM, whereas the HGMB1-NSC group received $2 \times 10^{5}$ NSCs preconditioned with $1 \mathrm{ng} / \mathrm{ml}$ HMGB1 in $3 \mu \mathrm{l}$ DMEM. To maximize the engraftment of the NSCs injected into the spinal cord, the needle remained in the spinal cord for 10 min after injection. All transplantation procedures were performed under sterile condition.

Evaluation of behavioral and sensory functions. The locomotor recovery in rats after SCI was assessed according to the Basso, Beattie and Bresnahan (BBB) scale (15). Prior to undergoing $\mathrm{SCI}$, rats ( $\mathrm{n}=6$ from each experimental group) were exposed to the testing system for 3 days, and their bladders were emptied before testing. For examination, rats were placed individually in an open field with a non-slippery surface in a large Plexiglas ${ }^{\circledR}$ field $(150 \mathrm{~cm}$ long $\times 150 \mathrm{~cm}$ wide $\times 30 \mathrm{~cm}$ high $)$ on days 1, 7, 14, 21 and 28 post-SCI. Locomotor recovery was assessed and scored by two independent examiners blinded to the experimental groups.

Mechanical allodynia was measured by two independent researchers blinded to the experimental conditions on days 1 , 7, 14, 21 and 28 post-SCI using an automatic von Frey apparatus (Dynamic Plantar Aesthesiometer; Ugo Basile SRL). Rats were placed individually in a plastic cage with a wire net floor and were left for $30 \mathrm{~min}$ to acclimate. The withdrawal response threshold ( $26 \mathrm{~g})$ was performed five times, and the values reported are the mean of these five measures.

The thermal sensitivity of the plantar hind paws was assessed by two independent investigators using the cold plate test (Cold/Hot Plate Analgesia Meter; Columbus Instruments), which was reported previously (16). The temperature of the cold plate was maintained at $5^{\circ} \mathrm{C}$ and the cut-off latency was $30 \mathrm{sec}$. The latency (in sec) to withdrawal from the cold plate was recorded along with other behaviors indicating attendance to the stimulus, including licking, looking at the affected paw sniffing or attacking the stimulus. The remaining five latencies were averaged for each rat.

Hematoxylin and eosin $(H \& E)$ and Nissl staining. On day 21 post-SCI, rats ( $\mathrm{n}=4$ from each experimental group) were 
A
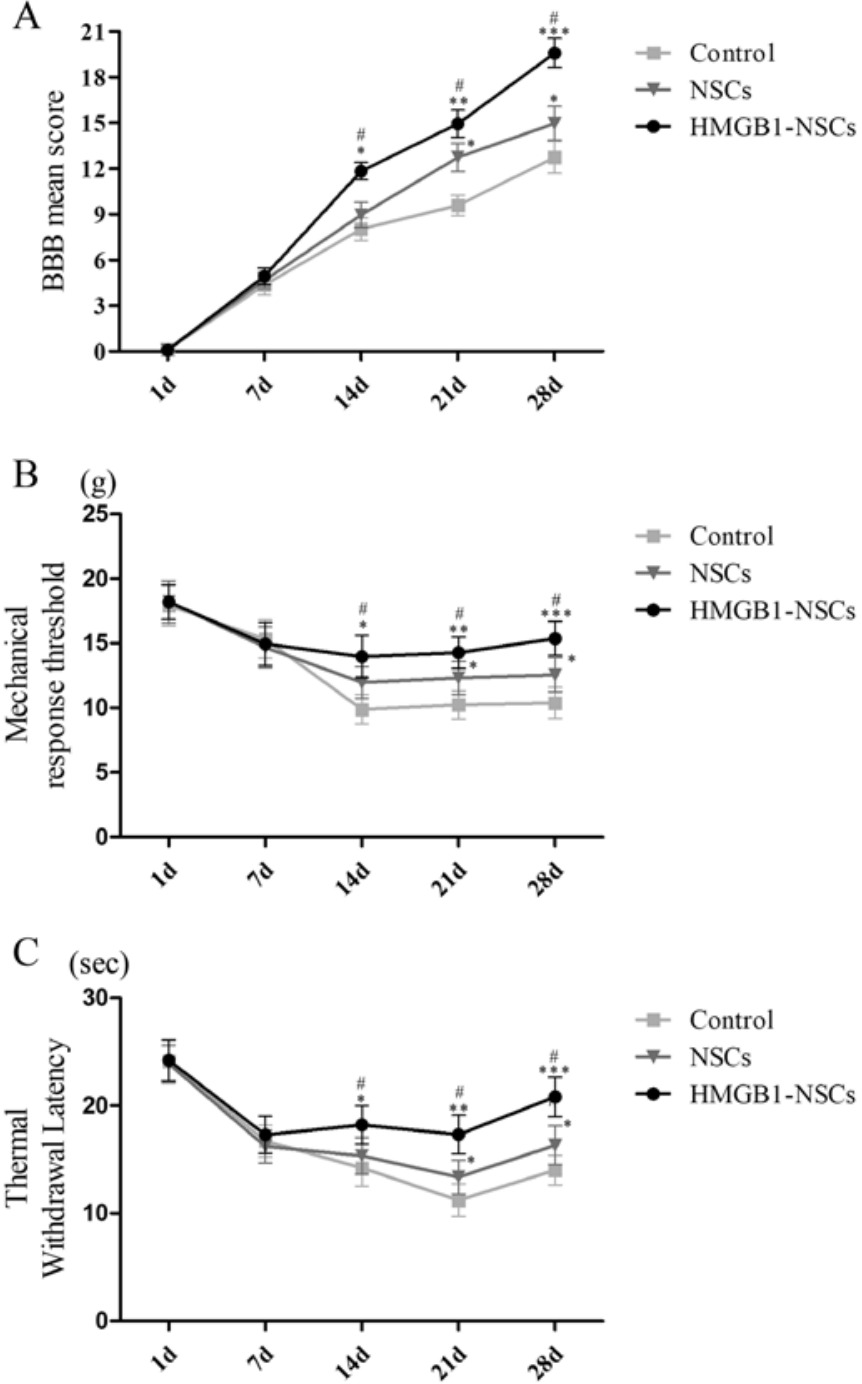

Figure 1. Transplantation of HMGB1-preconditioned NSCs promotes functional recovery after SCI. (A) Highest improvement in motor function of the hindlimb was observed in the HMGB1-NSC group compared with the control and NSC groups from day 14 post-SCI. (B) SCI-induced hypersensitivity to mechanical stimulation was significantly alleviated in the HMGB1-NSC group compared with the control and NSC groups from day 14 post-SCI. (C) SCI-induced hypersensitivity to cold stimulation was significantly attenuated in the HMGB1-NSC group compared with the control and NSC groups from day 14 post-SCI. $n=6$ from each experimental group. Data were analyzed using two-way ANOVA followed by Tukey's post hoc test ${ }^{*} \mathrm{P}<0.05,{ }^{* * *} \mathrm{P}<0.01,{ }^{* * *} \mathrm{P}<0.001$ vs. control group; ${ }^{*} \mathrm{P}<0.05$ vs. NSC group. NSC, neuronal stem cells; HMGB1; high mobility group box-1; SCI, spinal cord injury; BBB, Basso, Beattie and Bresnahan scale.

anesthetized using $2 \%$ isoflurane/air mixture $(1-2 \mathrm{~L} / \mathrm{min})$ and perfused through the ascending aorta with $0.9 \%$ saline followed by $400 \mathrm{ml} \mathrm{4 \%}$ paraformaldehyde in phosphate buffered solution (PBS, $0.1 \mathrm{M}, \mathrm{pH} ~ 7.4$ ). The T10 spinal cord segment $(1 \mathrm{~cm}, 0.5 \mathrm{~cm}$ either side from the injury epicenter) containing the injury epicenter and surrounding uninjured tissues were dissected, post-fixed for $48 \mathrm{~h}$ in $4 \%$ paraformaldehyde at $4^{\circ} \mathrm{C}$, embedded in paraffin, and cut into 5- $\mu \mathrm{m}$ sections using a vibratome. Sections were stained with a standard H\&E or Nissl staining procedure $(17,18)$ and visualized using a light microscope (Leica Microsystems $\mathrm{GmbH}$ ). The sagittal plane sections with every fifth interval were prepared. Five sections were stained, analyzed, and the cross-sectional areas were calculated and reported as the average of four independent measurements. All measurements were made by a technician who was blinded to the experiment groups.

Immunostaining. For immunostaining, single NSCs $\left(1 \times 10^{5} / \mathrm{ml}\right)$ seeded in poly-L-ornithine (PLO)-pre-coated confocal culture dishes or frozen sections $\left(-20^{\circ} \mathrm{C}\right)$ from spinal cords ( $\mathrm{n}=4$ from each experimental group) were fixed using 4\% paraformaldehyde in $0.01 \mathrm{M}$ PBS at room temperature for $1 \mathrm{~h}$. Then, the samples were blocked with $5 \%$ v/v FBS supplemented with $0.5 \%$ Triton X-100 (Beyotime Institute of Biotechnology) in $0.01 \mathrm{M}$ PBS at $37^{\circ} \mathrm{C}$ for $30 \mathrm{~min}$. Samples were incubated with rabbit monoclonal anti- $\beta$ III-tubulin primary antibody (clone no. Tuj-1; 1:200; cat. no. 5568; Cell Signaling Technology, Inc.) overnight at $4^{\circ} \mathrm{C}$. Then, samples were incubated with a Alexa Fluor ${ }^{\circledR} 555$ or 488 -conjugated secondary antibody (1:100; cat. nos. A0453 and A0423; Beyotime Institute of Biotechnology) at room temperature for $2 \mathrm{~h}$. Cell nuclei were counterstained with DAPI (Sigma-Aldrich; Merck KGaA) for $10 \mathrm{~min}$ at room temperature. Samples were mounted onto glass slides and images were captured using a confocal microscope (LSM780; Carl Zeiss AG) and examined by Zen 2011 software (Carl Zeiss AG). The sagittal plane sections at every fifth interval were prepared. Five sections were stained, analyzed, and the cross-sectional areas were calculated and reported as the average of four independent measurements. All measurements were made by a technician who was blinded to the experiment groups.

Western blotting. The T10 spinal cord segments $(\mathrm{n}=3$ from each experimental group; $1 \mathrm{~cm}, 0.5 \mathrm{~cm}$ either side from the injury epicenter) containing the injury epicenter and surrounding uninjured tissues or NSCs after preconditioning with $1 \mathrm{ng} / \mathrm{ml}$ HMGB1 were isolated and collected immediately from rats following decapitation on day 21 after SCI. The samples were homogenized with RIPA (Beyotime Institute of Biotechnology) supplemented with protease inhibitor cocktail (Roche Diagnostics). Then, the protein concentration was measured using a BCA Protein Assay kit (Beyotime Institute of Biotechnology). Proteins (40 $\mu \mathrm{g} / \mathrm{lane}$ ) were separated by SDS-PAGE on a $10 \%$ gel under reducing conditions and electroblotted to polyvinylidene difluoride membranes (Roche Diagnostics). The membranes were blocked in 5\% BSA (Beyotime Institute of Biotechnology) in TBS with Tween-20 (TBST) at room temperature for $2 \mathrm{~h}$. Subsequently, the membranes were cut out at different parts according to a pre-stained protein molecular ladder (cat. no. 26616; Thermo Fisher Scientific, Inc.) to allow separate detection of proteins migrating at the same distance, and were incubated with anti- $\beta$ III-tubulin rabbit monoclonal antibody $(1: 1,000$; cat. no. 5568; Cell Signaling Technology, Inc.), anti-ERK rabbit monoclonal antibody (1:1,000; cat. no. 4695; Cell Signaling Technology, Inc.), anti-phosphorylated (p)-ERK rabbit polyclonal antibody (1:1,000; cat. no. 4370; Cell Signaling Technology, Inc.) and anti-GAPDH mouse monoclonal antibody (1:1,000; cat. no. AF0006; Beyotime Institute of Biotechnology) overnight at $4^{\circ} \mathrm{C}$. Membranes were washed 3 times with TBST, following which they were incubated in the corresponding horseradish peroxidase-conjugated goat 

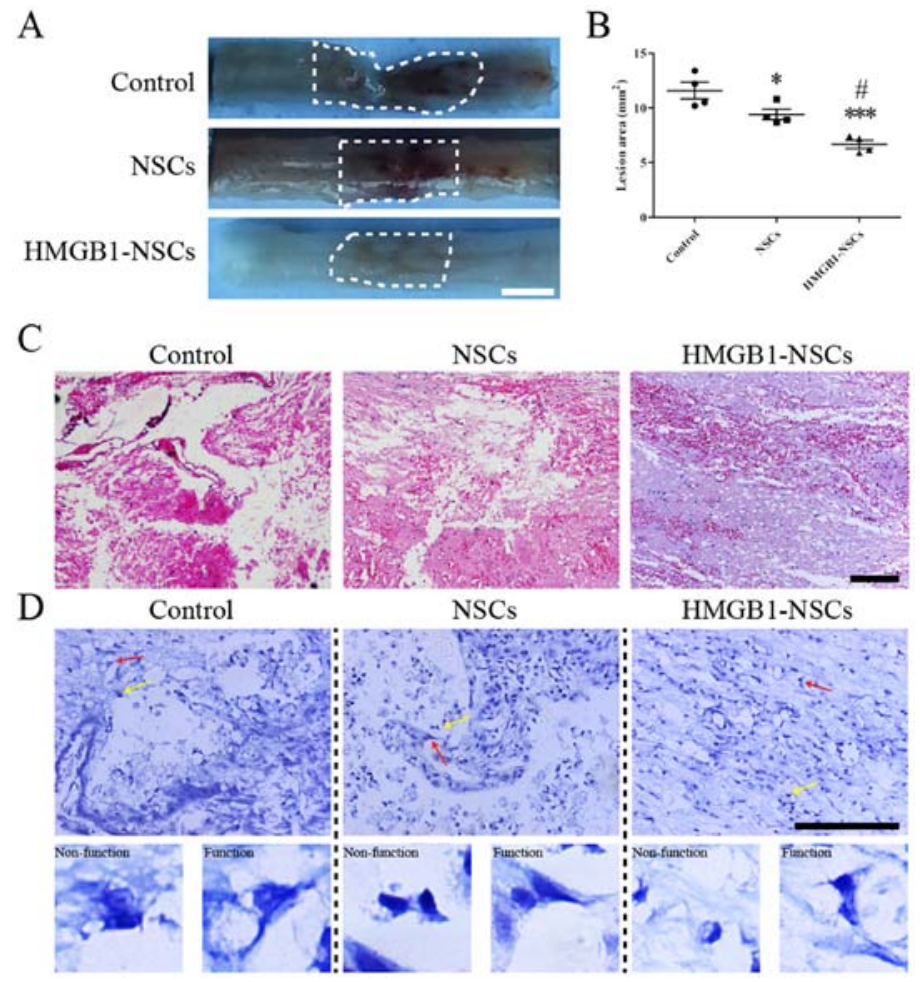

Figure 2. Histological assessment of the epicenter of the injured spinal cord on day 21 post-SCI. (A) Representative images of injured spinal cords obtained from rats in the control, NSC and HMGB1-NSC groups on day 21 post-SCI. (B) Summarized data showing the lesion area among control, NSC and HMGB1-NSC groups. (C) Hematoxylin and eosin staining showed spinal cord atrophy in the control, NSC and HMGB1-NSC groups on day 14 post-SCI. (D) Nissl staining showed residual neurons at the epicenter of injured spinal cords in the control, NSC and HMGB1-NSC groups on day 21 post-SCI. The yellow arrow indicates a non-functional Nissl body, the red arrow indicates a functional Nissl body; insets are zoomed in areas of the Nissl bodies. Scale bar, $100 \mu \mathrm{m}$. $\mathrm{n}=4$ from each experimental group. Data were analyzed using one-way ANOVA followed by Tukey's post hoc test. ${ }^{*} \mathrm{P}<0.05,{ }^{* * *} \mathrm{P}<0.01$ vs. control group; ${ }^{*} \mathrm{P}<0.05$ vs. NSC group. NSC, neuronal stem cells; HMGB1; high mobility group box-1; SCI, spinal cord injury.

anti-rabbit or goat anti-mouse secondary $\operatorname{IgG}$ antibody $(1: 5,000$; cat. nos. A0208 and A0216, respectively; Beyotime Institute of Biotechnology) at room temperature for $2 \mathrm{~h}$. Subsequently, bands were visualized with a chemiluminescence reagent kit (Beyotime Institute of Biotechnology) using a ChemiDoc ${ }^{\text {TM }}$ XRS+ imaging system (Bio-Rad Laboratories, Inc.), and semi-quantified using Image Lab software (version 2.0.1; Bio-Rad Laboratories, Inc.). GAPDH was used as the internal control to normalize the expression level of each protein.

NSC differentiation. For differentiation, NSCs $\left(1 \times 10^{5} / \mathrm{ml}\right)$ were seeded in $10 \mu \mathrm{g} / \mathrm{ml}$ PLO-pre-coated confocal culture dishes and incubated in DMEM/F12 medium supplemented with B27 and $1 \%$ GlutaMAX ${ }^{\mathrm{TM}}$ with or without $1 \mathrm{ng} / \mathrm{ml} \mathrm{HMGB1 \text {for }}$ 10 days at $37^{\circ} \mathrm{C}$ with $5 \% \mathrm{CO}_{2}$, as recommended. At the end of the initial 10-day culture, the ERK antagonist U0126 $(10 \mu \mathrm{M}$; cat. no. S1901; Beyotime Institute of Biotechnology) was dissolved in DMSO and added to the differentiation medium for $24 \mathrm{~h}$ at $37^{\circ} \mathrm{C}$ in a humidified atmosphere with $5 \% \mathrm{CO}_{2}$. Half of the volume of culture medium was changed every 3 days.

Statistical analysis. All data are presented as the mean \pm SEM, and statistical analyses were performed using SPSS version 19.0 (IBM Corp.). Two-tailed Student t-tests were used for comparisons between two groups. Multiple comparisons were performed using ANOVA followed by Tukey's post hoc test for multiple pair-wise comparisons. $\mathrm{P}<0.05$ was considered to indicate a statistically significant difference.

\section{Results}

Transplantation of HMGB1-preconditioned NSCs promotes functional recovery after $S C I$ in rats. To investigate the effect of transplanting HMGB1-preconditioned NSCs on functional recovery after SCI in rats, BBB scores, mechanical hypersensitivity and cold plate tests were performed on days 1, 7, 14, 21 and 28 post-SCI in the three groups: Control, NSCs and HMGB1-NSCs. The results indicated that rats in the HMGB1-NSC group showed the highest improvement in locomotor recovery compared with the control and NSC groups on days 14, 21 and 28 (Fig. 1A). Meanwhile, in the NSC group the outcome of locomotor recovery was improved compared with the control group on days 21 and 28 (Fig. 1A). Additionally, the score for mechanical hypersensitivity was the highest in the HMGB1-NSC group compared with the control and NSC groups on days 14, 21 and 28 (Fig. 1B). Additionally, the mechanical stimulation score was higher in the NSC group compared with the control group on days 14, 21 and 28 (Fig. 1B). In addition, the thermal withdrawal latency score was highest in the HMGB1-NSC group compared with the control and NSC groups on days 14, 21 and 28 (Fig. 1C). Furthermore, this score was higher in the NSC group compared with the control group on days 21 and 28 (Fig. 1C). Collectively, based on these data related to functional recovery, the time point of day 21 was significant for all three groups. Therefore, we performed the sampling measurements on day 21 . 
A

DAPI / BIII-tubulin
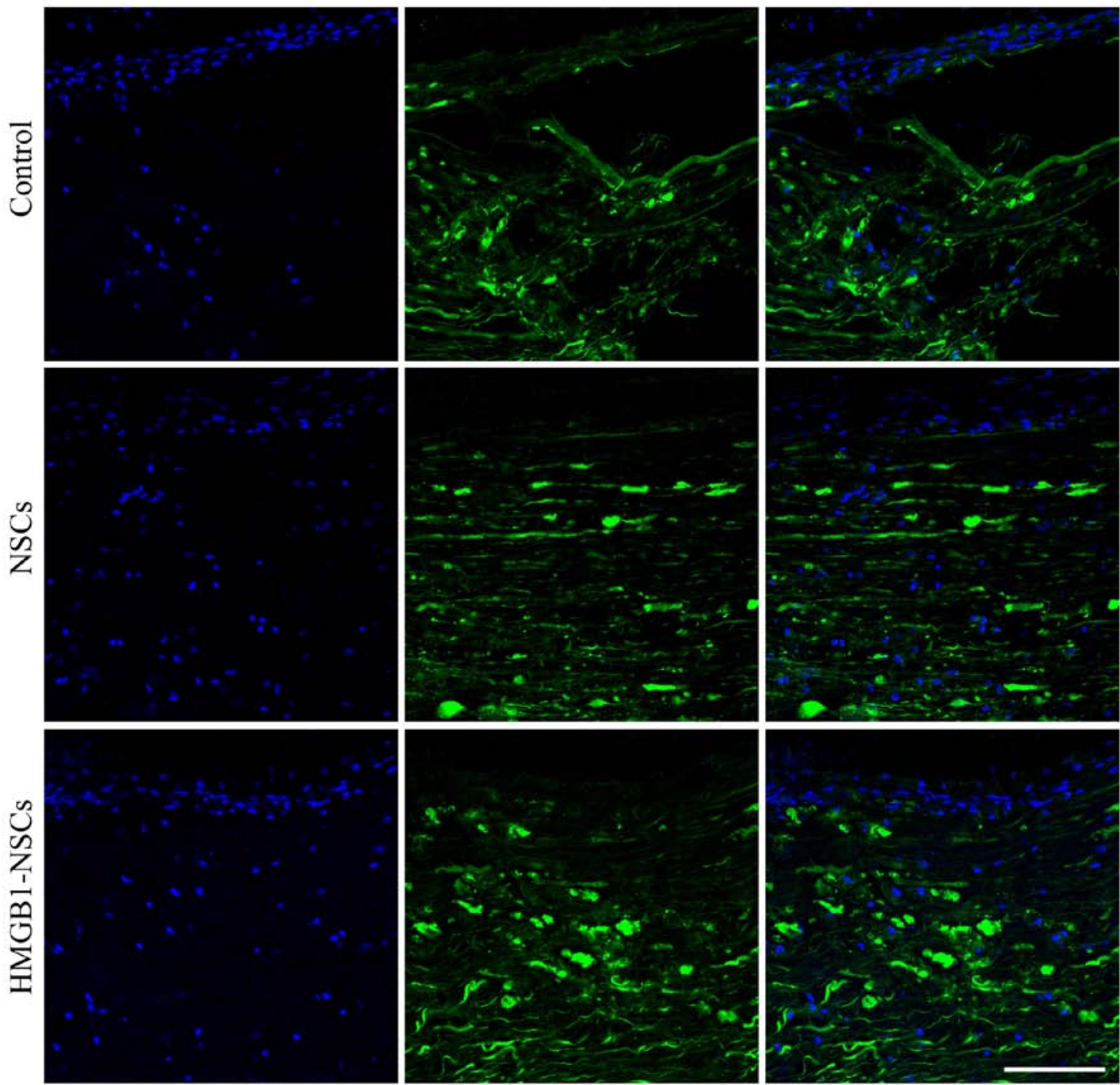

B

$\mathrm{C}$

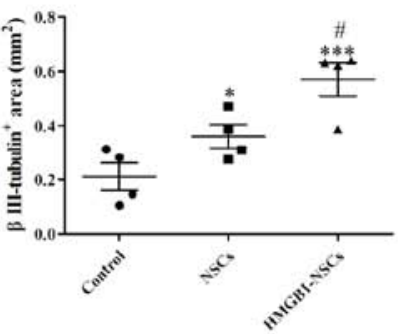

$\mathrm{D}$

$\beta$ III-tubulin
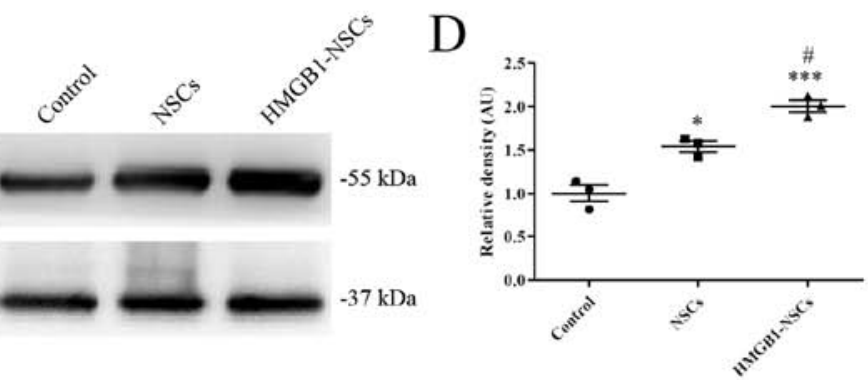

Figure 3. Transplantation of HMGB1-preconditioned NSCs increases the number of neurons at the epicenter of the injured spinal cord. (A) Immunostaining showed the expression of $\beta$ III-tubulin (green) at the epicenter of the injured spinal cord on day 21 post-SCI. Cell nuclei were stained blue with DAPI. Scale bar, $20 \mu \mathrm{m}$. (B) Representative graph of $\beta I I I-$ tubulin $^{+}$areas in different groups on day $21 . \mathrm{n}=4$ from each experimental group. (C) Western blotting was used to determine the expression of $\beta$ III-tubulin in the control, NSC and HMGB1-NSC groups. (D) Semi-quantification of the expression of $\beta$ III-tubulin in the injured spinal cords in the control, NSC and HMGB1-NSC groups. $n=3$ from each experimental group. Data were analyzed using one-way ANOVA followed by Tukey's post hoc test. ${ }^{*} \mathrm{P}<0.05,{ }^{* * *} \mathrm{P}<0.01$ vs. control group; ${ }^{*} \mathrm{P}<0.05$ vs. NSC group. NSC, neuronal stem cells; HMGB1; high mobility group box-1; SCI, spinal cord injury.

Transplantation of HMGB1-preconditioned NSCs enhances histological benefit after SCI in rats. To investigate why functional recovery was evidently improved in rats receiving transplantation of NSCs preconditioned with $1 \mathrm{ng} / \mathrm{ml} \mathrm{HMGB1}$, H\&E and Nissl staining were performed to explore histological changes on day 21 post-SCI. The data showed that the lesion area in the HMGB1-NSC group was significantly decreased compared with the NSC and control groups (Fig. 2A and B). The lesion region in the NSC group was smaller compared with the control group (Fig. 2A and B). Subsequently, H\&E staining showed the fewest number of basophilic nuclei and the smallest areas of spared tissue on day 21 post-SCI 


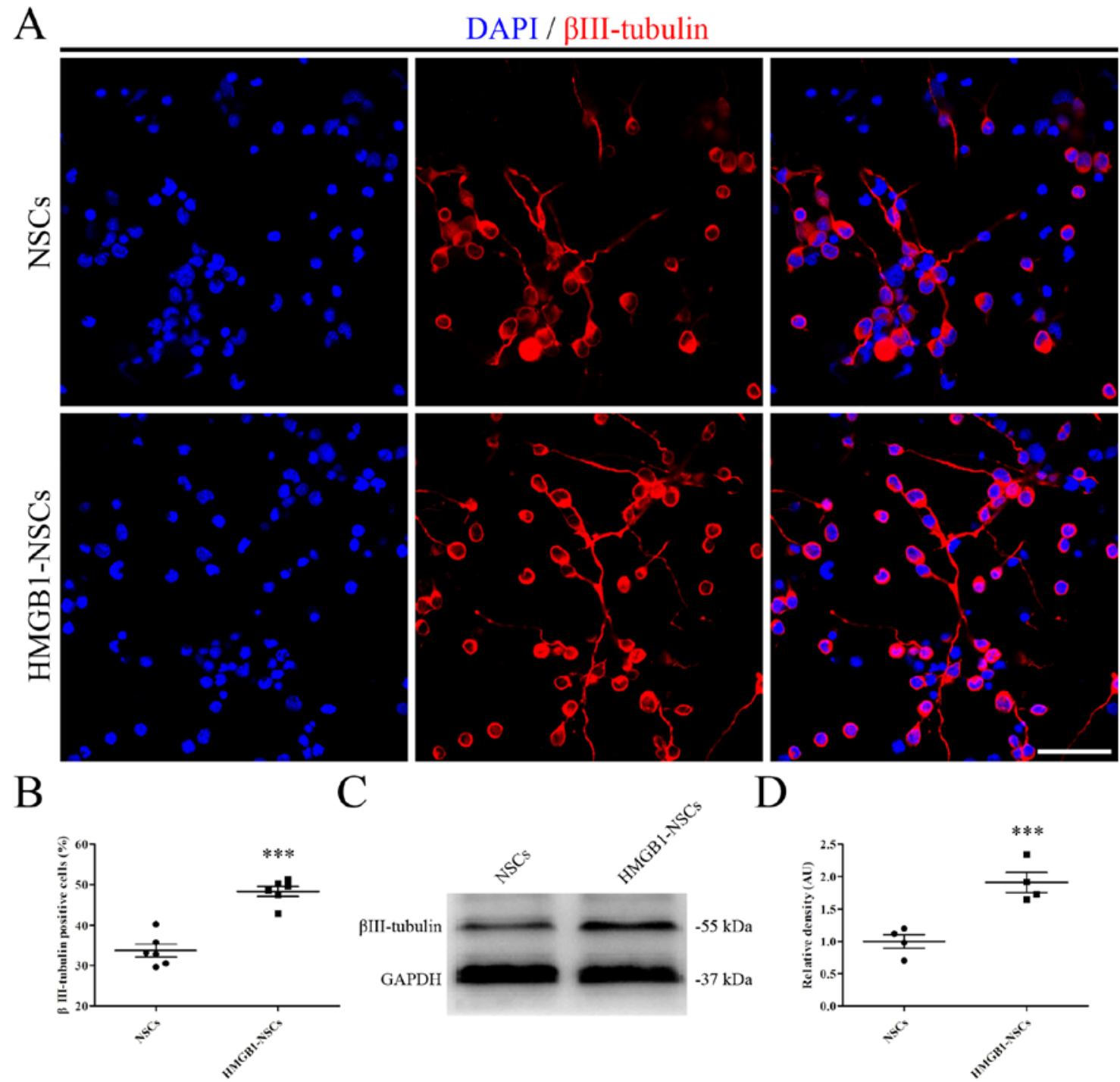

Figure 4. HMGB1 facilitates neuronal differentiation. (A) Immunostaining was used to determine the number of $\beta$ III-tubulin ${ }^{+}$cells (red) in NSC and HMGB1-NSC groups. Cell nuclei were stained blue with DAPI. Scale bar, $20 \mu \mathrm{m}$. (B) Quantitative results from immunostaining. $\mathrm{n}=6$ from each experimental group. (C) Western blotting was used to determine the expression of $\beta$ III-tubulin in NSC and HMGB1-NSC groups. Bands were cut out from different parts of the same gel according to a pre-stained protein ladder and analyzed using Image Lab software for relative density and expression was normalized to GAPDH (D) Semi-quantitative analysis of $\beta$ III-tubulin expression. $\mathrm{n}=4$ from each experimental group. Data were analyzed using Student's t-test. ${ }^{* * *} \mathrm{P}<0.01$ vs. NSC group. NSC, neuronal stem cells; HMGB1; high mobility group box-1.

(Fig. 2C). Moreover, functional Nissl bodies were visible in the HMGB1-NSC group (Fig. 2D).

Transplantation of HMGB1-preconditioned NSCs facilitates neuronal survival post-SCI in rats. Furthermore, immunostaining was performed to evaluate the number of residual neurons at the epicenter of the injured spinal cord at day 21 post-SCI. The images indicated that the $\beta$ III-tubulin ${ }^{+}$area in the HMGB1-NSC group was larger compared with the NSC group (Fig. 3A and B). Similarly, the $\beta$ III-tubulin ${ }^{+}$ area in the NSC group was larger compared with the control group (Fig. 3A and B). Next, $\beta$ III-tubulin expression was determined via western blotting and the results were consistent with the results obtained from immunostaining (Fig. 3C and D).

ERK signaling pathway plays a role in NSC differentiation induced by HMGB1. To further elucidate how
HMGB1-preconditioned NSC transplantation facilitates residual neuronal survival in vivo, NSCs were incubated in differentiation medium with or without $1 \mathrm{ng} / \mathrm{ml}$ HMGB1 for 10 days in vitro. NSCs treated with $1 \mathrm{ng} / \mathrm{ml}$ HMGB1 promoted neuronal differentiation, as indicated by the increase in the neuronal marker $\beta$ III-tubulin (Fig. 4A and B). Next, the results were confirmed by western blotting (Fig. 4C and D). Furthermore, to uncover the potential mechanism underlying the ability of HMGB1 treatment to promote neuronal differentiation, the expression of p-ERK was determined by immunoblotting. Treatment with HMGB1 led to a significant increase in p-ERK expression in NSCs (Fig. 5A and B). In addition, a specific ERK antagonist, $10 \mu \mathrm{M}$ U0126, was used to determine the role of ERK signaling in HMGB1-mediated NSC differentiation. Although the frequency of $\beta$ III-tubulin ${ }^{+}$cells in the HMGB1-NSC group was higher compared with the NSC group, U0126 reversed this effect (Fig. 5C and D). 
A

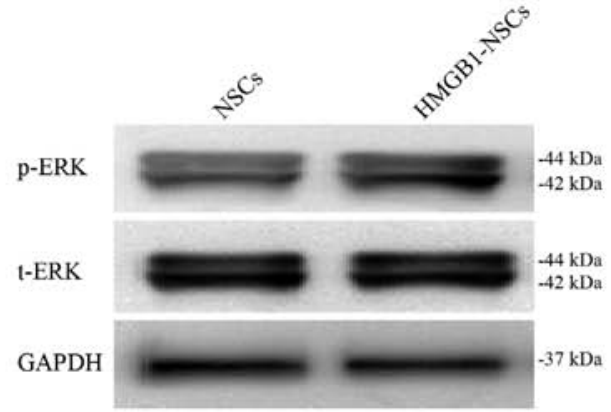

$\mathrm{C}$
B

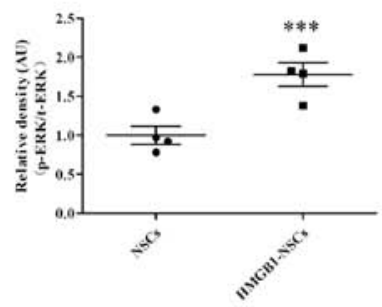

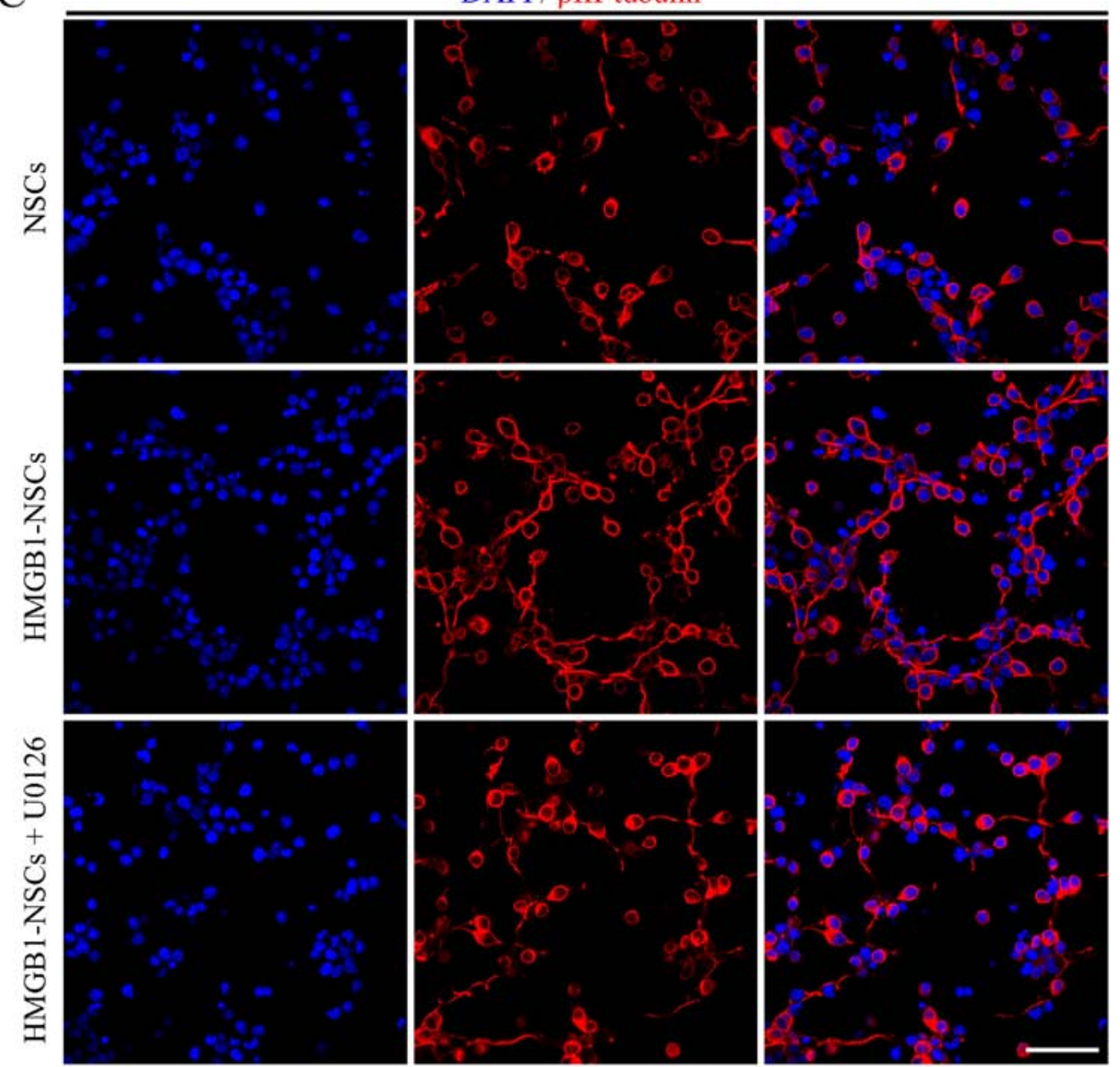

$\mathrm{D}$

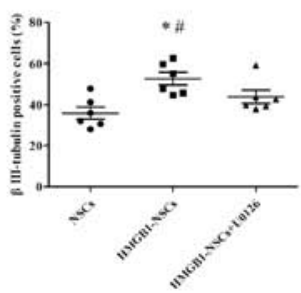

Figure 5. ERK signaling pathway plays an important role in NSC differentiation induced by HMGB1. (A) Western blotting was used to determine the expression levels of p-ERK in NSC and HMGB1-NSC groups. Bands were cropped from different parts of the same gel according to a pre-stained protein ladder and analyzed using the Image Lab software for relative density and expression was normalized to GAPDH. (B) Semi-quantitative analysis of p-ERK expression. $\mathrm{n}=4$ from each experimental group. ${ }^{* * *} \mathrm{P}<0.01$ vs. NSC group. (C) Immunostaining showed $\beta I I I-$ tubulin ${ }^{+}$cells (red) in NSC, HMGB1-NSC and HMGB1-NSC + U0126 groups. Cell nuclei were stained blue with DAPI. Scale bar, $20 \mu \mathrm{m}$. (D) Quantitative results from immunostaining. $\mathrm{n}=6$ from each experimental group. Data were analyzed using one-way ANOVA followed by Tukey's post hoc test. "P $<0.05$ vs. NSC group; ${ }^{*} \mathrm{P}<0.05$ vs. HMGB1-NSC $+\mathrm{U} 0126$ group. NSC, neuronal stem cells; HMGB1; high mobility group box-1; p-, phosphorylated; t-, total.

\section{Discussion}

The present study verified the hypothesis that transplantation of HMGB1-preconditioned NSCs facilitated the functional recovery of SCI in rats, as indicated by the decreased lesion area and increased neuronal survival. Meanwhile, the results also indicated that treatment with $1 \mathrm{ng} / \mathrm{ml}$ HMGB1 enhanced NSC differentiation into neurons in vitro, and the ERK signaling 
pathway played a role in this process. Mechanistically, the present results indicated that the HMGB1 preconditioning method is a feasible approach for stem cell replacement therapy.

HMGB1 has been demonstrated to help facilitate angiogenesis and neurogenesis after central nervous system injury. HMGB1 was first reported on in 1973 as a non-histone chromosomal protein (19) and is commonly known as a danger signal- or damage-associated molecular pattern (20). Recently, a study revealed that transplantation of NSCs prior to anti-HMGB1 antibody administration alleviated blood-spinal cord barrier disruption and edema formation, and increased the number of neurites from spared axons and survival of host neurons, resulting in functional recovery (21). Meanwhile, a study also indicated that the HMGB1/RAGE signaling pathway played a central role in facilitating endogenous NSC differentiation into mature neurons (22). Furthermore, a previous study demonstrated that $1 \mathrm{ng} / \mathrm{ml}$ HMGB1 not only promoted NSC proliferation, but also facilitated NSC migration in vitro (13). The present study confirmed that HMGB1 promoted NSC differentiation into neurons after SCI in rats, which is consistent with previous studies $(22,23)$. In addition, another study reported that HMGB1 promoted angiogenesis and neurogenesis in the late phase of intracerebral hemorrhage by upregulating vascular endothelial growth factor and nerve growth factor (24).

It has been proposed that the HMGB1/RAGE axis may play a significant role in promoting NSC differentiation into neurons following HMGB1 treatment. A previous study demonstrated that $1 \mathrm{ng} / \mathrm{ml}$ HMGB1 activated RAGE to augmentNSC proliferation and migration(13). Differentiation is one of the main features of NSCs. In the present study, the results also indicated that the HMGB1/RAGE signaling pathway increased the number of residual neurons at the epicenter of the injured spinal cord to promote functional recovery. Meanwhile, the present results also demonstrated that ERK expression in NSCs was upregulated after HMGB1 treatment, which is consistent with a previous finding that $1 \mathrm{ng} / \mathrm{ml}$ HMGB1 enhanced neurulation via the MAPK signaling pathway (25). Moreover, a previous study also reported that the $\mathrm{HMGB} 1 / \mathrm{RAGE} / \mathrm{NF}-\kappa \mathrm{B}$ signaling pathway played an essential role in promoting differentiation of hippocampal neural progenitor cells into neurons in Alzheimer's disease (26). Whether the RAGE/NF- $\kappa \mathrm{B}$ axis is involved in the process of HMGB1-facilitated NSC differentiation needs further investigation in the future. Meanwhile, the present data indicated that engraftment of HMGB1-preconditioned NSCs increased the number of neurons at the epicenter and facilitated neuronal differentiation following SCI. The increased number of neurons might partly derive from transplanted NSCs. In addition, the engrafted NSCs might also promote the survival of local neurons due to the secretion of neurotrophic and growth factors (NT-3, GDNF and BDNF) from engrafted NSCs, as described in a previous study (2). Herein, our next study will focus on determining the proportion of local surviving neurons and those differentiation from engrafted NSCs at the epicenter of the injured spinal cord.

However, there are limitations of the present study that need to be investigated or improved upon in future studies, these include: i) The method of administrating
HMGB1-preconditioned NSCs, such as via intravenous injection; ii) the ideal time point of HMGB1-preconditioned NSC engraftment; iii) the transfection rate of NSCs/HMGB1-preconditioned NSCs injected into the spinal cord; iv) axon formation between caudal and rostral sides of the injury site; v) a more appropriate time point for the sampling measurements (immunostaining and western blotting), instead of day 21 ; vi) the use of a non-model control that the present study lacked; vii) more NSC-specific markers and differentiation markers should be analyzed via immunostaining to provide more evidence for HMGB1-facilitaed NSC differentiation. However, the present study provides evidence that the use of HMGB1 is a feasible approach to optimize cell replacement therapy when transplanting NSCs after SCI.

In summary, the present study indicated that transplantation of NSCs preconditioned with $1 \mathrm{ng} / \mathrm{ml} \mathrm{HMGB} 1$ could significantly improve functional recovery by decreasing injured spinal cord atrophy and increasing the number of $\beta$ III-tubulin ${ }^{+}$cells at the epicenter of the injured spinal cord, which provides a possible strategy for NSC transplantation in the treatment of SCI.

\section{Acknowledgements}

The authors would like to thank Dr Hongfei Ge (Department of Neurosurgery, Southwest Hospital, Third Military Medical University, Chongiqng, China) for his technical support.

\section{Funding}

This work was supported by The National Natural Science Foundation of China (grant no. 81972068).

\section{Availability of data and materials}

All data generated or analyzed during this study are included in this published article.

\section{Authors' contributions}

MYL and JHZ participated in the design of the study. XX, LZ and XY performed the experiments. XXC, ZFC, CXW and $\mathrm{YX}$ analyzed the data. All authors read and approved the final manuscript.

\section{Ethics approval and consent to participate}

The present study was approved by The Third Military Medical University Ethics Committee (approval no. SYXK 2012-0002) and followed the regulations of the China Laboratory Animal Guidelines.

\section{Patient consent for publication}

Not applicable.

\section{Competing interests}

The authors declare that they have no competing interests. 


\section{References}

1. Li X, Liu D, Xiao Z, Zhao Y, Han S, Chen B and Dai J: Scaffold-facilitated locomotor improvement post complete spinal cord injury: Motor axon regeneration versus endogenous neuronal relay formation. Biomaterials 197: 20-31, 2019.

2. Fan WL, Liu P, Wang G,Pu JG, Xue X andZhao JH: Transplantation of hypoxic preconditioned neural stem cells benefits functional recovery via enhancing neurotrophic secretion after spinal cord injury in rats. J Cell Biochem 119: 4339-4351, 2018.

3. Vismara I, Papa S, Rossi F, Forloni G and Veglianese P: Current options for cell therapy in spinal cord injury. Trends Mol Med 23: 831-849, 2017.

4. Upadhyay G, Shankar S and Srivastava RK: Stem cells in neurological disorders: Emerging therapy with stunning hopes. Mol Neurobiol 52: 610-625, 2015.

5. Assinck P, Duncan GJ, Hilton BJ, Plemel JR and Tetzlaff W: Cell transplantation therapy for spinal cord injury. Nat Neurosci 20 : 637-647, 2017.

6. Jin H, Zhang YT, Yang Y, Wen LY, Wang JH, Xu HY, Lai BQ, Feng B, Che MT, Qiu XC, et al: Electroacupuncture facilitates the integration of neural stem cell-derived neural network with transected rat spinal cord. Stem Cell Reports 12: 274-289, 2019.

7. Yousefifard M, Rahimi-Movaghar V, Nasirinezhad F, Baikpour M, Safari S, Saadat S, Moghadas Jafari A, Asady H, Razavi Tousi SMT and Hosseini M: Neural stem/progenitor cell transplantation for spinal cord injury treatment; A systematic review and meta-analysis. Neuroscience 322: 377-397, 2016.

8. Karova K, Wainwright JV, Machova-Urdzikova L, Pisal RV, Schmidt M, Jendelova P and Jhanwar-Uniyal M: Transplantation of neural precursors generated from spinal progenitor cells reduces inflammation in spinal cord injury via NF- $\beta B$ pathway inhibition. J Neuroinflammation 16: 12, 2019.

9. Kojima K, Miyoshi H, Nagoshi N, Kohyama J, Itakura G, Kawabata S, Ozaki M, Iida T, Sugai K, Ito S, et al: Selective ablation of tumorigenic cells following human induced pluripotent stem cell-derived neural stem/progenitor cell transplantation in spinal cord injury. Stem Cells Transl Med 8: 260-270, 2019.

10. Hooshmand MJ, Sontag CJ, Uchida N, Tamaki S, Anderson AJ and Cummings BJ: Analysis of host-mediated repair mechanisms after human CNS-stem cell transplantation for spinal cord injury: Correlation of engraftment with recovery. PLoS One 4: e5871, 2009.

11. Babu H, Cheung G, Kettenmann H, Palmer TD and Kempermann G: Enriched monolayer precursor cell cultures from micro-dissected adult mouse dentate gyrus yield functional granule cell-like neurons. PLoS One 2: e388, 2007.

12. Ronaghi M, Erceg S, Moreno-Manzano V and Stojkovic M: Challenges of stem cell therapy for spinal cord injury: Human embryonic stem cells, endogenous neural stem cells, or induced pluripotent stem cells? Stem Cells 28: 93-99, 2010

13. Xue X, Chen X, Fan W, Wang G, Zhang L, Chen Z, Liu P, Liu M and Zhao J: High-mobility group box 1 facilitates migration of neural stem cells via receptor for advanced glycation end products signaling pathway. Sci Rep 8: 4513, 2018.

14. Ge H, Tan L, Wu P, Yin Y, Liu X, Meng H, Cui G, Wu N, Lin J, $\mathrm{Hu} \mathrm{R}$ and Feng H: Poly-L-ornithine promotes preferred differentiation of neural stem/progenitor cells via ERK signalling pathway. Sci Rep 5: 15535, 2015
15. Basso DM, Beattie MS and Bresnahan JC: Graded histological and locomotor outcomes after spinal cord contusion using the NYU weight-drop device versus transection. Exp Neurol 139: 244-256, 1996.

16. Jurga AM, Rojewska E, Piotrowska A, Makuch W, Pilat D, Przewlocka B and Mika J: Blockade of toll-like receptors (TLR2, TLR4) attenuates pain and potentiates buprenorphine analgesia in a rat neuropathic pain model. Neural Plast 2016: 5238730, 2016.

17. Hu R, Zhou J, Luo C, Lin J, Wang X, Li X, Bian X, Li Y, Wan Q, Yu Y and Feng H: Glial scar and neuroregeneration: Histological, functional, and magnetic resonance imaging analysis in chronic spinal cord injury. J Neurosurg Spine 13: 169-180, 2010.

18. Liu H, Zhou J, Gu L and Zuo Y: The change of HCN1/HCN2 mRNA expression in peripheral nerve after chronic constriction injury induced neuropathy followed by pulsed electromagnetic field therapy. Oncotarget 8: 1110-1116, 2017.

19. Paudel YN, Semple BD, Jones NC, Othman I and Shaikh MF: High mobility group box 1 (HMGB1) as a novel frontier in epileptogenesis: From pathogenesis to therapeutic approaches. J Neurochem 151: 542-557, 2019.

20. Hei Y, Chen R, Yi X, Long Q, Gao D and Liu W: HMGB1 neutralization attenuates hippocampal neuronal death and cognitive impairment in rats with chronic cerebral hypoperfusion via suppressing inflammatory responses and oxidative stress. Neuroscience 383: 150-159, 2018.

21. Uezono N, Zhu Y, Fujimoto Y, Yasui T, Matsuda T, Nakajo M, Abematsu M, Setoguchi T, Mori S, Takahashi HK, et al: Prior treatment with anti-high mobility group box-1 antibody boosts human neural stem cell transplantation-mediated functional recovery after spinal cord injury. Stem Cells 36: 737-750, 2018.

22. Wang H, Mei X, Cao Y, Liu C, Zhao Z, Guo Z, Bi Y, Shen Z, Yuan Y, Guo Y et al: HMGB1/advanced glycation end products (RAGE) does not aggravate inflammation but promote endogenous neural stem cells differentiation in spinal cord injury. Sci Rep 7: 10332, 2017.

23. Tirone M, Tran NL, Ceriotti C, Gorzanelli A, Canepari M, Bottinelli R, Raucci A, Di Maggio S, Santiago C, Mellado M, et al: High mobility group box 1 orchestrates tissue regeneration via CXCR4. J Exp Med 215: 303-318, 2018.

24. Lei C, Lin S, Zhang C, Tao W, Dong W, Hao Z, Liu M and Wu B: Effects of high-mobility group box 1 on cerebral angiogenesis and neurogenesis after intracerebral hemorrhage. Neuroscience 229: $12-19,2013$

25. Wang L, Yu L, Zhang T, Wang L, Leng Z, Guan Y and Wang X: HMGB1 enhances embryonic neural stem cell proliferation by activating the MAPK signaling pathway. Biotechnol Lett 36: 1631-1639, 2014.

26. Meneghini V, Bortolotto V, Francese MT, Dellarole A, Carraro L, Terzieva $S$ and Grilli M: High-mobility group box-1 protein and $\beta$-amyloid oligomers promote neuronal differentiation of adult hippocampal neural progenitors via receptor for advanced glycation end products/nuclear factor- $\mathrm{KB}$ axis: Relevance for Alzheimer's disease. J Neurosci 33: 6047-6059, 2013.

This work is licensed under a Creative Commons Attribution-NonCommercial-NoDerivatives 4.0 International (CC BY-NC-ND 4.0) License. 